\title{
Hausärztliche Einstellungen, Vorgehensweisen und Herausforderungen bei der Unterstützung pflegender Angehöriger - Ergebnisse einer Befragung von Allgemeinmediziner*innen
}

\section{General practitioners, attitudes, procedures and challenges towards supporting family caregivers - results of a survey of primary care physicians}

\section{다 (i) $(2)$}

Autoren

Julian Wangler, Michael Jansky

Institut

Zentrum für Allgemeinmedizin und Geriatrie,

Universitätsmedizin Mainz

Schlüsselwörter

Pflegende Angehörige, Hausarzt, Identifizierung,

Belastungen, Bedürfnisse, Betreuung

Key words

caregivers, general practitioner, identification, strain, needs, care, support

online publiziert 18.11 .2021

Bibliografie

Dtsch Med Wochenschr 2022; 147: e1-e12

DOI 10.1055/a-1671-8621

ISSN 0012-0472

(C) 2021. The Author(s).

This is an open access article published by Thieme under the terms of the Creative Commons Attribution-NonDerivative-NonCommercial License, permitting copying and reproduction so long as the original work is given appropriate credit. Contents may not be used for commecial purposes, or adapted, remixed, transformed or built upon. (https://creativecommons.org/licenses/by-nc-nd/4.0/)

Georg Thieme Verlag KG, Rüdigerstraße 14,

70469 Stuttgart, Germany

Korrespondenzadresse

Dr. Julian Wangler

Wissenschaftlicher Mitarbeiter | Zentrum für

Allgemeinmedizin und Geriatrie

Universitätsmedizin Mainz, Am Pulverturm 13, 55131 Mainz, Deutschland

Tel.: $+49 / 6131 / 178712$

Fax: $+49 / 6131 / 176601$

julian.wangler@unimedizin-mainz.de,www.unimedizin-

mainz.de/allgemeinmedizin

Zusätzliches Material finden Sie unter

https://doi.org/10.1055/a-1671-8621

\section{ZUSAMMENFASSUNG}

Hintergrund Hausärzt*innen gelten als gut geeignet, wenn es darum geht, die Informations- und Betreuungsbedürfnisse pflegender Angehöriger zu adressieren. Ziel der vorliegenden Studie ist es, eine Bestandsaufnahme zu leisten, wie Hausärzt*innen ihre Möglichkeiten, pflegende Personen zu unterstützen, einschätzen, welche Schwerpunkte sie setzen und inwieweit sie Herausforderungen erleben.

Methoden Mittels Online-Befragung mit schriftlich-postalischem Anschreiben wurden zwischen Februar und Juni 2021 insgesamt 3556 Hausärzt*innen in Baden-Württemberg, Hessen und Rheinland-Pfalz befragt. Aufgrund des explorativen Ansatzes der Studie erfolgte ausschließlich eine deskriptive Datenanalyse.

Ergebnisse $68 \%$ der Befragten haben häufig mit pflegenden Angehören zu tun; $77 \%$ halten die Hausarztpraxis als primäre Anlaufstelle für Pflegende und die Pflegekoordination für gut geeignet. Verbreitet kommen Betreuungssituationen zustande, indem Angehörige auf Hausärzt*innen zugehen (89\%). Häufige Inhalte betreffen eine Verschlechterung der Pflegesituation (75\%) und eine Veränderung des Pflegebedarfs (84\%); Beratungsgespräche in der Anfangsphase der Pflege sind seltener (40\%). Bei der Wahrnehmung von Bedürfnissen Pflegender und der Prioritätensetzung zeigen sich Unterschiede zwischen urbanen und ländlichen Ärzt*innen. Landärzt*innen gewichten eine proaktive und psychosoziale Betreuung stärker, wohingegen Ärzt*innen in städtischen Regionen vermehrt auf das Facharzt- und Hilfsnetzwerk setzen. Hausärzt*innen erleben diverse Herausforderungen bei der Betreuung Pflegender, darunter die zeitnahe Organisation passender Entlastungsangebote (87\%), die Vermittlung zu geeigneten Hilfsangeboten (79\%) oder die frühzeitige Identifizierung informeller Pflegepersonen (59\%).

Schlussfolgerung Die Hausarztpraxis kann eine zentrale Rolle bei der Unterstützung pflegender Angehöriger spielen. Pflegende sollten frühzeitig vom Praxisteam angesprochen und konsequent an Hilfs- und Unterstützungsangebote verwiesen werden. Zur effektiven Unterstützung einer gelingenden Pflege ist es wichtig, in der triadischen Konstellation die 
Bedürfnisse Pflegender und Gepflegter gleichermaßen zu berücksichtigen.

\section{ABSTRACT}

Background General practitioners are considered to be well suited when it comes to addressing the information and care needs of family caregivers. The aim of the present study is to examine how general practitioners assess their possibilities to support caregivers, what priorities they set and to what extent they experience challenges.

Methods and participants In the course of an online survey with a postal cover letter, a total of 3,556 GPs in in BadenWürttemberg, Hesse and Rhineland-Palatinate were interviewed between February and June 2021. Due to the exploratory approach of the study, only a descriptive data analysis was carried out.

Results $68 \%$ of the GPs surveyed often deal with family caregivers in everyday practice; $77 \%$ consider the GP's office to be well suited as the primary point of contact for family caregivers and care coordination. Often it is caregiving relatives who ask the GP about the issue of care (89\%). Frequent contents concern a deterioration in the care situation (75\%) and a change in the need for care (84\%); consultations in the initial phase of care are less common (40\%). There are differences between urban and rural doctors in the perception of the needs of caregivers and the setting of priorities. Rural doctors give more weight to proactive and psychosocial care, whereas doctors in urban regions rely on the specialist and support network. GPs experience various challenges while supporting caregivers, including the timely organization of suitable relief offers ( $87 \%$ ), the referral to suitable offers of help $(79 \%)$ or the early identification of informal caregivers (59\%).

Discussion GPs can play a central role in supporting family caregivers. A crucial prerequisite for this is that family caregivers are recognized and involved at an early stage. Consistent references to offers of help make it easier for family caregivers to organize care and to receive (psychosocial) support. In addition, it is important that GPs take into account the needs, desires and stresses of both caregivers and those being cared for.

\section{Hintergrund}

In der EU-27 sind heute über $20 \%$ der Bevölkerung 65 Jahre oder älter [1, 2]. Daraus ergibt sich ein wachsender Bedarf nach Pflege und Betreuung. Für Deutschland zeigt sich dieser Bedarf anhand von ca. 4,1 Millionen als formell pflegebedürftig eingestuften Menschen [3]. Rechnet man informell-unentgeltliche Unterstützungs- und Pflegetätigkeiten hinzu, erhöht sich diese Zahl auf ca. 5,5 Millionen [4, 5].

Informelle Pflege wird überwiegend im häuslichen Umfeld durch private Pflegepersonen erbracht, die bei der Betreuung und Versorgung von ihnen nahestehenden hilfsbedürftigen Menschen einen beträchtlichen Teil der Fürsorge tragen [6-8]. Repräsentativdaten zufolge unterstützen mehr als $17 \%$ der 40 - bis 85 -Jährigen mindestens eine Person regelmäßig bei der Bewältigung des Alltags; hiervon leistet gut jeder Dritte Pflege im engeren Sinne [9, 10].

Obwohl Untersuchungen nachweisen konnten, dass eine pflegende Tätigkeit mit subjektivem Sinnerleben einhergehen kann $[11,12]$, ist sie aufgrund der physischen und mentalen Belastung mit einem höheren Gesundheitsrisiko assoziiert [10, 13-15]. Entsprechend verbreitet sind unter Pflegenden Beschwerden wie Erschöpfungszustände und depressive Symptome [8, 16-18]. Hat eine Auseinandersetzung mit den Folgen der Erkrankung im Vorfeld nicht stattgefunden und wurden Vorsorgemaßnahmen nicht ergriffen, kommt es nicht selten zu Überforderungssituationen [15].

Um solche Krisen zu vermeiden und die Resilienzfähigkeit Pflegender zu fördern, sind diverse Unterstützungsangebote etabliert worden. In Deutschland zählt hierzu die Tätigkeit von Pflegestützpunkten, ambulanten psychiatrischen Diensten und DemenzNetzwerken. Allerdings zeigen Arbeiten, dass solche Angebote nur von einem Teil pflegender Personen genutzt werden [19, 20].
Aufgrund der kontinuierlichen Betreuung und langjähriger Kenntnis gelten Hausärzt*innen als gut geeignet, um häusliche Pflegesettings zu begleiten und gezielt auf pflegende Angehörige einzugehen [6, 21-23]. Abgesehen von der Diagnostik und Therapie gesundheitlicher Beschwerden können Hausärzt*innen im Gespräch mit Pflegenden informierend und beratend tätig werden, psychosoziale Unterstützung gewähren und sich ein Bild von den Pflegebedingungen verschaffen, sodass Bedarfe rechtzeitig adressiert werden können. Indem Hausärzt*innen auf Hilfs- und Beratungsangebote verweisen, können sie die Weichen für eine längerfristig gelingende Pflege stellen und Pflegenden dadurch Kompensations- und Entlastungsmöglichkeiten aufzeigen [19, 24].

Da Übergänge hin zu informellen Pflegepersonen oft fließend sind, kann es für das hausärztliche Team herausfordernd sein, pflegende Angehörige frühzeitig zu identifizieren [7, 22]. Schwierigkeiten ergeben sich, wenn die gepflegte Person nicht von der Praxis versorgt wird, in der der Angehörige selbst Patient ist [10]. Gerade in solchen Fällen kann es passieren, dass Allgemeinärzt*innen vorrangig die gepflegte Person wahrnehmen $[25,26]$ und Belastungen Angehöriger nicht ausreichend im Blick haben [21].

Eine Befragung der Kassenärztlichen Bundesvereinigung kommt zu dem Ergebnis, dass ca. 60 \% der pflegenden Angehörigen mit ihrem Hausarzt über ihr Pflegeengagement sprechen [23]. Mit Blick auf den Grad und die Art der hausärztlichen Unterstützung sowie die Betreuungsbedürfnisse Pflegender mangelt es gerade für den deutschsprachigen Raum an belastbaren Studien. Eine im Jahr 2020 durchgeführte Online-Befragung von insgesamt 612 pflegenden Personen, die in 17 Internetforen zum Thema Pflege geschaltet war [27], hat gezeigt, dass eine deutliche Mehrheit der Befragten die hausärztliche Kenntnis der Betreuungssituation, die Ansprechbarkeit bei pflegebezogenen Problemlagen und die Hinwendung zur Pflegeperson positiv beurteilt. 
Rund die Hälfte gibt an, Verweise auf Beratungs- und Hilfsangebote über den Hausarzt erhalten zu haben; ein ähnlich hoher Anteil bekundet, sich als pflegende Person rechtzeitig vom Hausarzt erkannt und einbezogen gefühlt zu haben. Die Ergebnisse einer Regressionsanalyse zeigen, dass die genannten Aspekte als Einflussfaktoren für die subjektiv erlebte Zufriedenheit mit der hausärztlichen Unterstützung sowie für das Gefühl, die Pflegesituation bewältigen zu können, bedeutsam sind.

Bislang fehlt es insbesondere für den deutschsprachigen Raum an Studien, die hausärztliche Einstellungs- und Handlungsmuster in Bezug auf die Zielgruppe pflegender Angehöriger, aber auch erlebte Herausforderungen breit beleuchten. Ziel der vorliegenden Studie ist es daher, eine grundlegende Bestandsaufnahme zu leisten, wie Hausärzt*innen ihre Möglichkeiten, pflegende Angehörige zu unterstützen, einschätzen und welche Schwerpunkte sie hierbei setzen.

\section{Erkenntnisinteresse}

Die Arbeit ist Teil einer explorativen, mehrteiligen Studie, die Perspektiven von pflegenden Angehörigen und Hausärzt*innen mit Blick auf die Unterstützungspotenziale der primärärztlichen Versorgung fokussiert. Indem unterschiedliche Perspektiven beider Gruppen betrachtet werden, sollen Ansatzpunkte einer Optimierung der hausärztlichen Versorgung abgeleitet werden. Das Erkenntnisinteresse der vorliegenden Teilstudie bündelt sich v. a. in folgenden Fragestellungen:

- Wie nehmen Hausärzt*innen ihre eigene Bedeutung als Ansprechpartner*innen für pflegende Personen wahr? Wie beurteilen sie ihre Möglichkeiten, Pflegende effektiv unterstützen zu können?

- Wie lassen sich Unterstützungs- und Betreuungskonstellationen im Umgang mit pflegenden Angehörigen beschreiben?

- Welche Betreuungsbedürfnisse nehmen Hausärzt*innen auf Seiten pflegender Angehöriger wahr? Welche Prioritäten setzen sie bei der Unterstützung?

- Inwieweit erleben Hausärzt*innen bei der Betreuung Pflegender Herausforderungen?

- Inwieweit machen Hausärzt*innen von Möglichkeiten zur Systematisierung und Effektivierung bei der Unterstützung Pflegender Gebrauch?

- Welche Verbesserungsansätze lassen sich für das hausärztliche Setting ableiten?

\section{Methodik}

\section{Studiendesign und Setting}

Bei der vorzustellenden Studie handelt es sich um eine eigenständige Untersuchung im weiteren inhaltlichen Kontext eines auf 3 Jahre geförderten Innovationsfonds-Modellprojektes zur ambulanten medizinisch-pflegerischen Demenzversorgung (DemStepCare, Proposal-ID: 01NVF18 027) [28, 29]. Dabei steht die Optimierung der hausarztbasierten Demenzversorgung im Zentrum. Das Modellprojekt DemStepCare wurde von der Ethikkommission der Landesärztekammer Rheinland-Pfalz genehmigt (Referenznummer: 2019-14427).
Der in diesem Beitrag behandelten Untersuchung ging die angesprochene Online-Befragung pflegender Angehöriger [27] voraus, die Betreuungsbedürfnisse und Erfahrungen in Bezug auf die hausärztliche Versorgung ermitteln sollte. Die vorliegende Arbeit sollte demgegenüber die Einstellungen und Erfahrungen von Hausärzt*innen mit Blick auf die Betreuung Pflegender erfassen und prüfen, inwieweit sich die Ergebnisse der vorangegangenen Befragung aus allgemeinärztlicher Perspektive bestätigen lassen. Die Wahl fiel auf eine Online-Befragung mit schriftlichpostalischem Anschreiben.

\section{Erhebungsinstrument und soziodemografische Variablen}

Es wurde ein eigenes Befragungsinstrument (s. Supplemental Material) entwickelt, das durch verschiedene Elemente abgestützt ist:

- Vorbereitungen der mehrteiligen Studienreihe (u. a. Gespräche mit pflegenden Angehörigen im Kontext von DemStepCare, Fokusgruppe mit acht Allgemeinmediziner*innen)

- Konzeption und Ergebnisse der Online-Befragung pflegender Angehöriger [27]

- Weitere Vorstudien der Autoren zur Demenzversorgung, in denen das Thema Pflege Berücksichtigung erfährt [z. B. 30]

- Allgemeine Literaturrecherche

Bei der Literaturrecherche wurden solche Arbeiten herangezogen, in denen pflegende Angehörige und ihre (potenzielle) Unterstützung im hausarztbasierten Setting im Zentrum der Betrachtung stehen [12, 17, 19, 22, 24, 26, 31], u. a. die Arbeiten von Geschke et al [19], Greenwood et al [22, 26] und Joling et al [17]. Zusätzlich wurde der Fragebogen vor dem Feldeinsatz einem Pretest unter 20 Hausärzt*innen unterzogen.

Ziel war es, den Fragebogen, der pflegenden Angehörigen vorgelegt wurde, teilweise zu adaptieren, sodass die Ergebnisse einander gegenübergestellt werden können. Als soziodemografische Merkmale wurden Alter, Geschlecht, Niederlassungsmodell, Patienten pro Quartal, Praxisumgebung und einschlägige Weiterbildungshintergründe erhoben.

Die Online-Befragung arbeitete mit Ausnahme mehrerer offener Fragen fast ausschließlich mit Pflichtfragen, ohne deren Beantwortung ein Fortschreiten im Fragebogen nicht möglich war. Der Grund hierfür war die Sicherstellung einer möglichst vollständigen Datenbasis, die bei klassischen schriftlich-postalischen Befragungen nicht immer gegeben ist.

\section{Rekrutierung und Teilnehmer}

Auf schriftlich-postalischem Weg zur Teilnahme an der anonymisierten Befragung eingeladen wurden zwischen Februar und Juni 2021 sämtliche 13170 als Behandler*innen aktive Hausärzt*innen in Baden-Württemberg (6664), Hessen (3839) und RheinlandPfalz (2667). Es handelte sich um ein einmaliges Anschreiben, in dem die zu befragenden Ärzt*innen unter anderem einen passwortgeschützten Zugang zur Online-Befragung mitgeteilt bekamen (keine Incentives). 


\section{Datenanalyse}

Die Daten wurden mittels SPSS 23.0 ausgewertet. Aufgrund des explorativen Ansatzes der Studie erfolgte ausschließlich eine deskriptive Datenanalyse. Die Likert-skalierten Skalenabstufungen wurden für die Ergebnisdarstellung teilweise zusammengefasst. Die Auswertung der offenen Fragen basiert auf einer Nachcodierung im Sinne der qualitativen Inhaltsanalyse [32]. Im Zuge der Sichtung der Antworten wurde ein Kategoriensystem erstellt, das mit Fortgang der Auswertung wiederholt geprüft und ggf. modifiziert wurde. Als Reporting Statement wurde STROBE herangezogen.

\section{Ergebnisse}

\section{Stichprobe}

Von den 3595 bearbeiteten Fragebögen gingen 3556 vollständig ausgefüllte Bögen in die Auswertung ein (Rücklauf: $27 \%$ ). Die Stichprobe ist wie folgt strukturiert:

- Geschlecht: $62 \%$ männlich, $38 \%$ weiblich

- Durchschnittsalter: 57 (Median: 56)

- Praxisumgebung: 50 \% mittel- und großstädtisch, 50 \% ländlichkleinstädtisch

- Praxisform: $58 \%$ Einzelpraxen, $42 \%$ Gemeinschaftspraxen

- Patienten pro Quartal: 28\% < 1500, 34\% 1501-2000, 38\% > 2000

- Weiterbildungshintergründe bzw. Zusatzbezeichnungen: $7 \%$ Palliativmedizin, 6 \% Sozialmedizin, 1 \% Psychotherapie, $18 \%$ Geriatrie

\section{Informations- und Beratungstätigkeit für pflegende Angehörige}

70 \% der Befragten vertreten die Auffassung, dass Hausärzt*innen als Ansprechpartner*innen für Fragen zur häuslichen Pflege von (sehr) großer Bedeutung sind. Wie eine offene Nachfrage zeigte, werden als Gründe hierfür die primärärztliche Rolle in Kombination mit langjähriger Kenntnis der Patient*innen und deren Angehörigen genannt. 68 \% geben an, im Praxisalltag (sehr) häufig mit pflegenden Angehörigen zu tun zu haben (32\% gelegentlich). Während 24 \% schätzen, dass die Zahl der betreuten Angehörigen in den zurückliegenden Jahren sehr stark zugenommen hat, gehen 52 \% von einer eher starken Zunahme aus (25\% gleichgeblieben).

Nach Angabe der Befragten ergibt sich das hausärztliche Gespräch mit pflegenden Personen vornehmlich dadurch, dass diese auf die Ärzt*innen zukommen, z. B. weil sie Rat möchten oder Fragen zur Pflegeorganisation haben (89\% sehr häufig/eher häufig). Demgegenüber bekunden $41 \%$, dass sich das initiale Gespräch häufig ergibt, indem die Ärzt*innen (proaktiv) auf die Pflegenden zugehen (z. B. Identifizierung von Pflegesituationen, Veranlassung eines Anamnesegesprächs/Assessments der Pflegesituation). $57 \%$ geben an, das Thema Pflege ergebe sich häufig beiläufig, beispielsweise über einen Check-up.

Den Angaben der Befragten zufolge ist die Situation verbreitet, dass die gepflegte Person langjährige/r Patient*in ist (82\% sehr häufig/eher häufig). 53 \% geben an, es komme häufig vor, dass die pflegende Person beständige/r Patient*in ist. Dominierende Inhalte des hausärztlichen Beratungsgesprächs sind die Veränderung des Pflegebedarfs (84\%), die Verschlechterung der Pflegesituation bzw. Anpassung an neue Gegebenheiten (75\%) sowie körperliche und/oder seelische Beschwerden (68\%). Seltener genannt werden die Veränderung der Beziehung zum Pflegebedürftigen (49\%) und der Pflegebeginn inklusive Vorbereitung und Planung einer häuslichen Pflege (40\%).

\section{Wahrnehmung und Prioritätensetzung in Bezug auf die Bedürfnisse pflegender Angehöriger}

- Tab. 1 stellt die von den Befragten bei Pflegenden wahrgenommenen Betreuungsbedürfnisse mit den Prioritäten der eigenen Betreuung gegenüber. Bei letzterer wurden die Befragten gebeten, maximal sechs Items auszuwählen, um vorrangige Schwerpunktsetzungen besser einkreisen zu können. Es zeigt sich, dass Hausärzt*innen auf Seiten pflegender Personen multidimensionale Bedürfnisse wahrnehmen, die sich allem voran in einer individuell passgenauen, kompetenten und persönlich zugewandten Betreuung bündeln. Während kleinstädtisch-ländliche Ärzt*innen die Wünsche und Erwartungen Pflegender stärker unter dem Gesichtspunkt der proaktiven, kollaborativen und psychosozialen Betreuung reflektieren, wozu durchaus auch Haubesuche gehören, sehen urban ansässige Ärzt*innen ihre Rolle stärker als Delegierer zu professionellen Hilfsangeboten und Fachärzt*innen. Ärzt*innen mit geriatrischer Weiterbildung fallen dadurch auf, dass sie erheblich öfter auf Beratungs- und Unterstützungsakteure verweisen oder dorthin vermitteln.

\section{Erlebte Herausforderungen}

Bei der Unterstützung Pflegender erleben Hausärzt*innen die Organisation zeitnaher Entlastungsangebote zur Abwendung krisenhafter Versorgungssituationen als anspruchsvoll; Vergleichbares gilt für die Benennung von und Vermittlung zu passenden Hilfsangeboten (vgl. > Tab. 2). Weitere Problematiken bestehen in der Bereitstellung ausreichender Zeitreserven für die oftmals komplexen Belastungen pflegender Angehöriger sowie die frühzeitige Identifizierung und Ansprache Pflegender. Einige der angeführten Herausforderungen werden von geriatrisch weitergebildeten Ärzt*innen in deutlich geringerem Maße wahrgenommen.

\section{Agieren in Hilfs- und Unterstützungsstrukturen}

Die Befragten wurden um eine Einschätzung gebeten, wie viele der in den letzten Jahren beratenen Angehörigen von ihnen auf konkrete Hilfsangebote aufmerksam gemacht bzw. dorthin verwiesen wurden. $16 \%$ der Befragten gehen davon aus, $60 \%$ oder mehr der Angehörigen auf solche Angebote aufmerksam gemacht zu haben; $32 \%$ gehen von rund der Hälfte aus (31\%: 33-50\%, 6\%: $33 \%$ oder weniger). Während $36 \%$ der urbanen Ärzt*innen angeben, $60 \%$ oder mehr der Angehörigen auf Unterstützungsstrukturen hingewiesen zu haben, sind dies unter Landärzt*innen $18 \%$. Unter geriatrisch weitergebildeten Ärzt*innen liegt dieser Anteil bei $46 \%$; bei den übrigen Ärzt*innen sind es $25 \%$. Von den Angeboten, auf die verwiesen wurde, werden am häufigsten neben Alten- und Pflegeheimen (72\%) lokale Ange- 


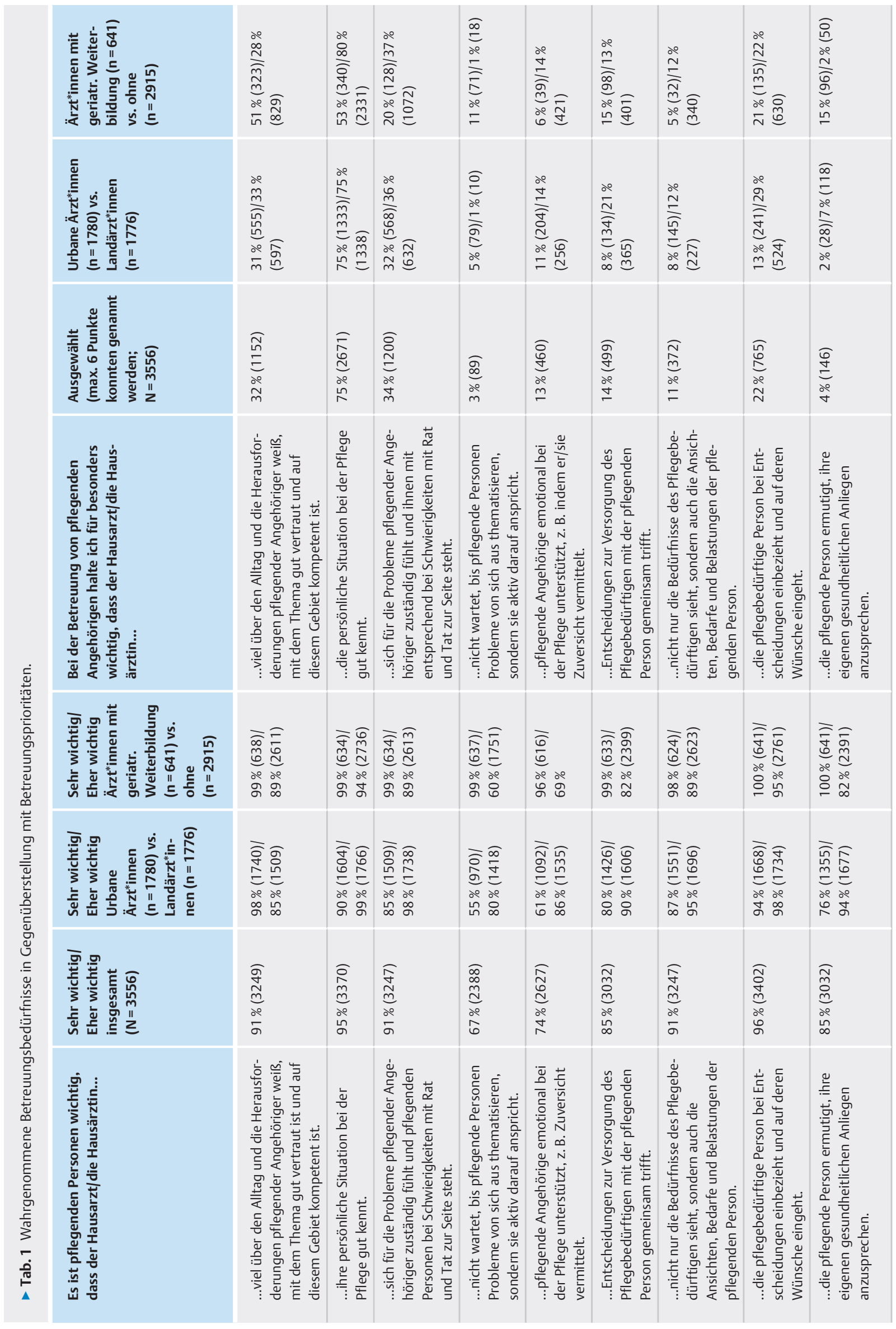




\begin{tabular}{|c|c|c|c|c|c|c|c|c|c|}
\hline 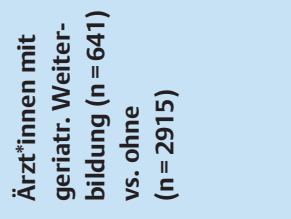 & 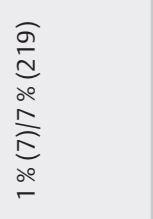 & 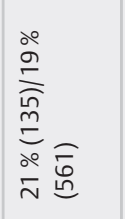 & 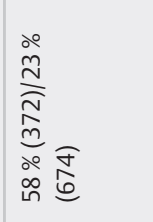 & 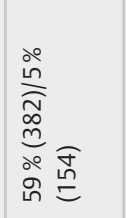 & 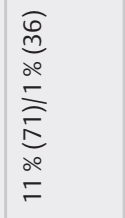 & 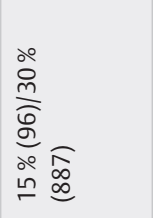 & 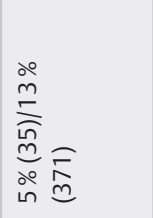 & 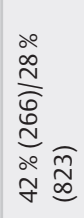 & 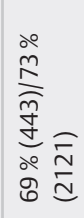 \\
\hline 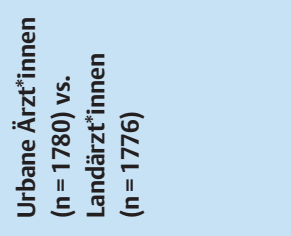 & 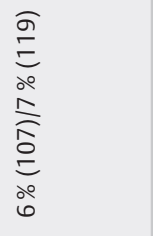 & 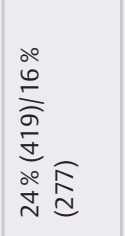 & 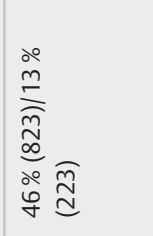 & 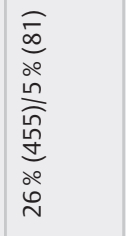 & 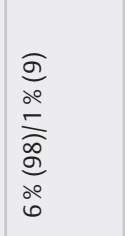 & 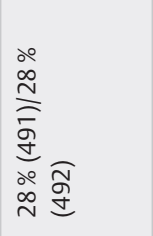 & 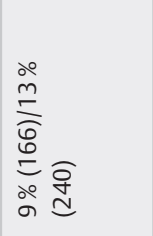 & 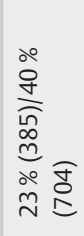 & 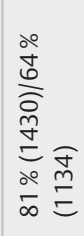 \\
\hline 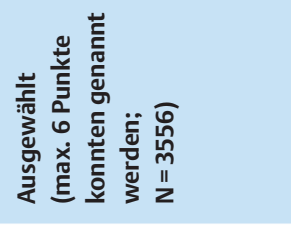 & & & 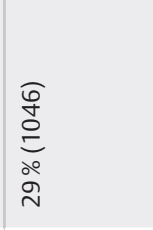 & 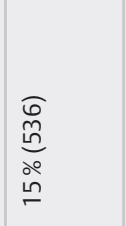 & $\begin{array}{l}\hat{\widehat{E}} \\
\stackrel{0}{=} \\
\stackrel{0}{m}\end{array}$ & $\begin{array}{l}\widehat{N} \\
\stackrel{\infty}{\circ} \\
\stackrel{0}{\infty} \\
\stackrel{\infty}{N}\end{array}$ & 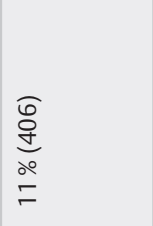 & $\begin{array}{l}\text { } \\
\stackrel{\circ}{0} \\
\stackrel{0}{\circ} \\
\stackrel{0}{m}\end{array}$ & 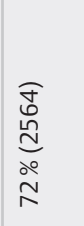 \\
\hline 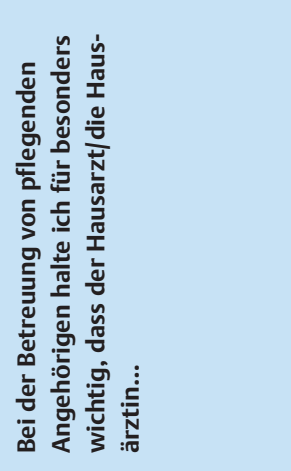 & 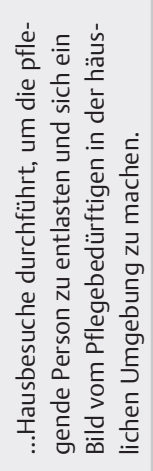 & 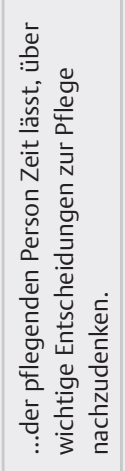 & 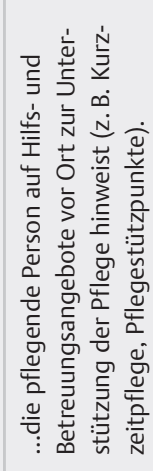 & 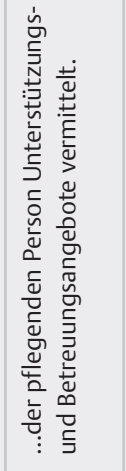 & 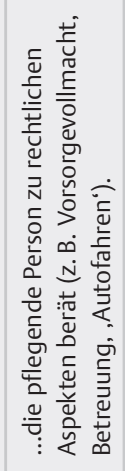 & 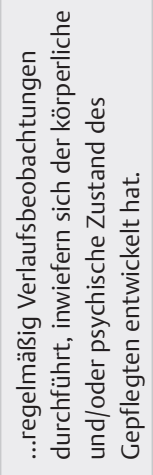 & 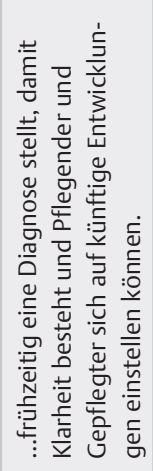 & 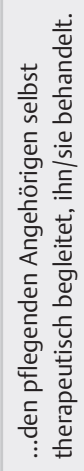 & 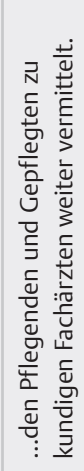 \\
\hline 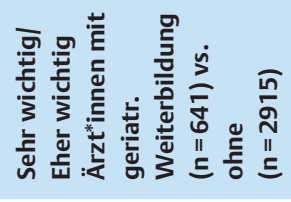 & 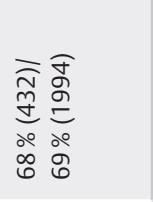 & 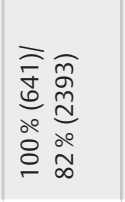 & 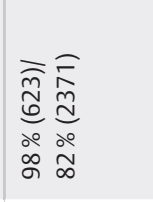 & 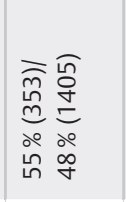 & 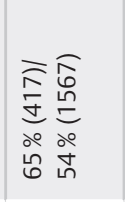 & 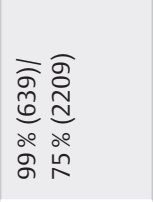 & 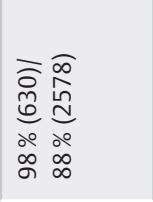 & 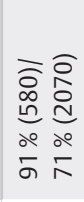 & 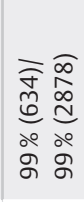 \\
\hline 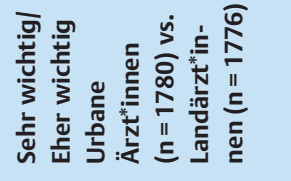 & 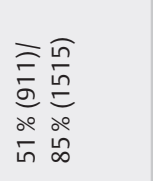 & 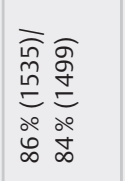 & 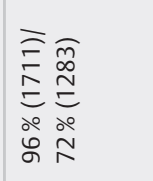 & 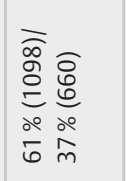 & 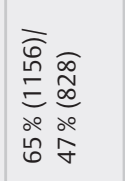 & 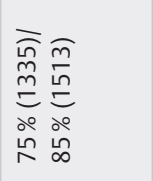 & 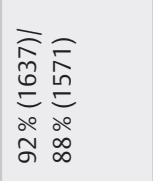 & 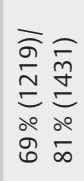 & 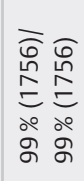 \\
\hline 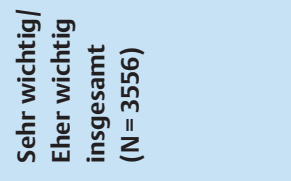 & 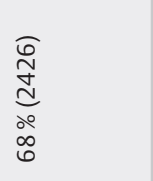 & 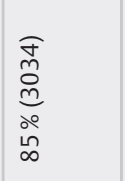 & 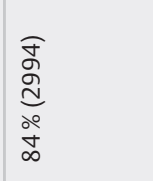 & 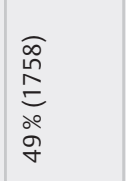 & 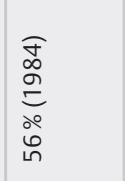 & $\begin{array}{l}\widehat{\infty} \\
\stackrel{\infty}{\infty} \\
\stackrel{0}{\sim} \\
\stackrel{0}{0} \\
\infty\end{array}$ & 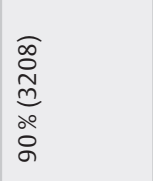 & 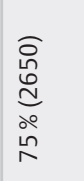 & 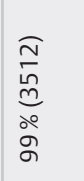 \\
\hline 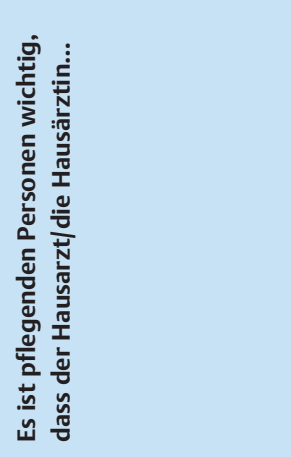 & 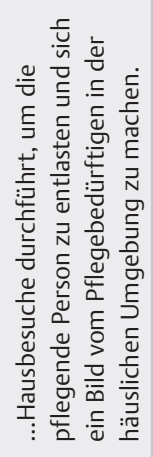 & 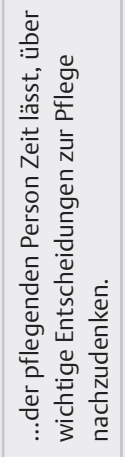 & 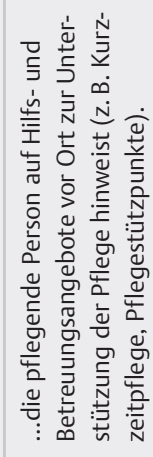 & 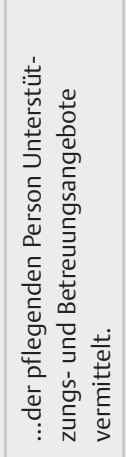 & 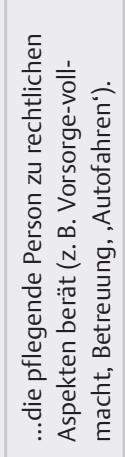 & 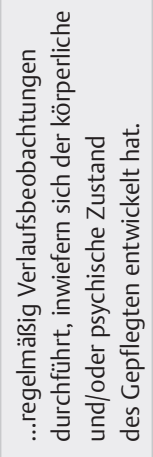 & 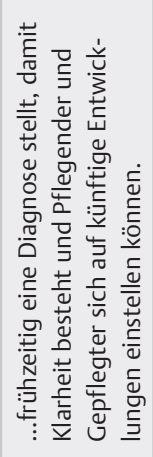 & 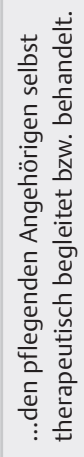 & 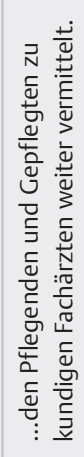 \\
\hline
\end{tabular}



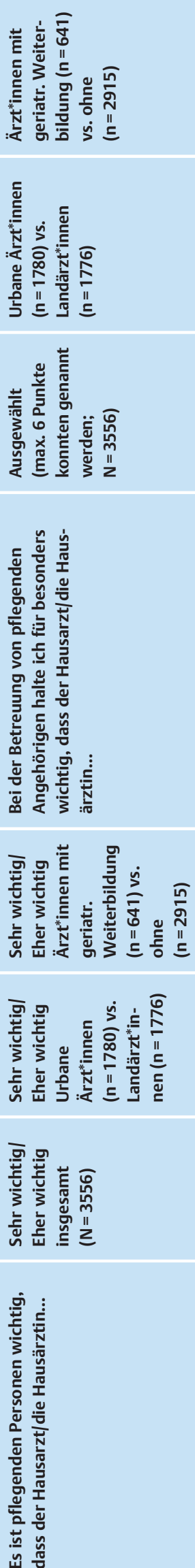
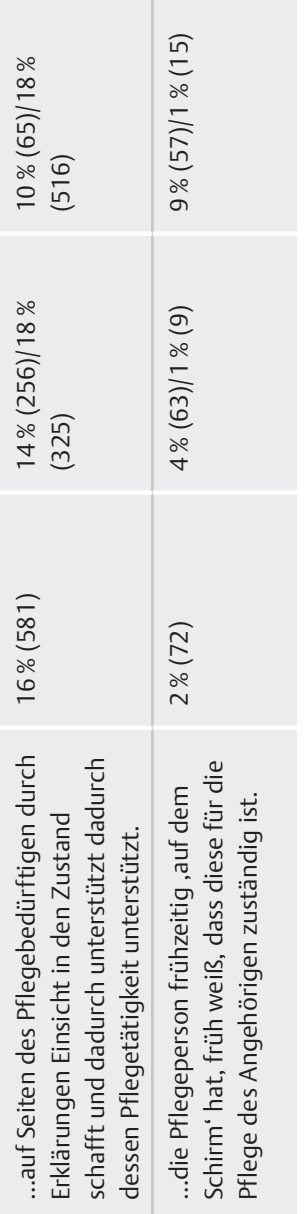

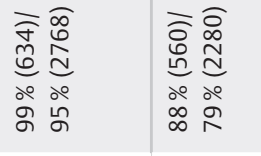

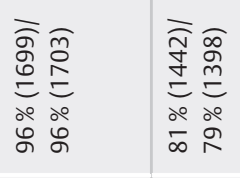

\begin{tabular}{|c|c|}
\hline $\begin{array}{l}\text { If } \\
\text { co } \\
\text { i̊ }\end{array}$ & $\begin{array}{c}\widehat{O} \\
\stackrel{o}{0} \\
\stackrel{0}{\sim} \\
\stackrel{0}{0} \\
\infty\end{array}$ \\
\hline 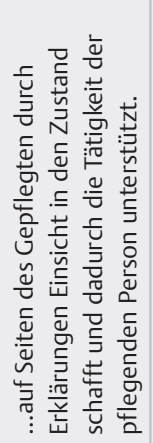 & 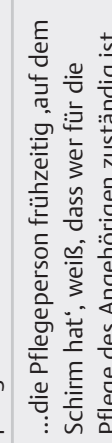 \\
\hline
\end{tabular}

bote zur Tages- oder Kurzzeitpflege (63\%) genannt, gefolgt von Pflegediensten bzw. Sozialstationen (57\%), Angeboten zur Unterstützung im Alltag (47\%) und Beratung zur Pflege sowie Pflegestützpunkte (40\%).

$40 \%$ der Ärzt*innen geben an, dass ihnen die S3-Leitlinie „Pflegende Angehörige“ (DEGAM) bekannt ist, bei denen eine hausarztkonforme, evidenzbasierte Betreuung dieser Zielgruppe im Mittelpunkt steht (60\% unbekannt). Hiervon bekunden $55 \%$, die Leitlinie häufig oder gelegentlich zu nutzen (44\% selten). Landärzte geben zu $29 \%$ an, die Leitlinie „Pflegende Angehörige“ zu kennen, urbane Ärzte zu 54\%.

$47 \%$ der Befragten haben innerhalb der eigenen Praxis Mitarbeiter*innen des nichtärztlichen Praxisteams, die die eigene Arbeit mit Blick auf die Betreuung pflegender Angehöriger regelmäßig unterstützen (z. B. aufgrund von vorangegangenen Fortbildungen). Aus dieser Gruppe geben $83 \%$ an, dass besagte Personen bei der Identifizierung Pflegender und deren Ansprache helfen; $44 \%$ nennen eine allgemeine Beratung, z. B. bei Fragen zur Pflegeorganisation. $80 \%$ der geriatrisch weitergebildeten Hausärzt*innen geben an, Praxispersonal bei der Betreuung pflegender Angehöriger einzubinden gegenüber 40 \% der übrigen Befragten.

Die weiteren Ergebnisse liefern Hinweise darauf, dass Hausärzt*innen, die das Personal im Themenzusammenhang integrieren, teils geringere Herausforderungen bei der Unterstützung Pflegender erleben. So empfinden es unter diesen kollaborativ arbeitenden Ärzt*innen 50 \% als große Herausforderung, Pflegende frühzeitig zu identifizieren, im Vergleich zu $66 \%$ der übrigen Ärzt*innen. 33 \% derjenigen, die bei der Unterstützung Pflegender auf ihr Praxispersonal zurückgreifen, erleben es als anspruchsvoll, einen kontinuierlichen Austausch mit Pflegepersonen sicherzustellen, dagegen $51 \%$ der sonstigen Ärzt*innen. $9 \%$ der kollaborativen Ärzt*innen erleben eine möglichst unverzögerte Einschätzung der Probleme und Bedürfnisse Pflegender als eher herausfordernd, hingegen $40 \%$ der anderen Befragten.

\section{Bilanzierung der Unterstützung pflegender Angehöriger und Optimierungsvorschläge}

47 \% der Befragten bilanzieren, dass es ihnen im Praxisalltag (sehr) gut möglich sei, auf die Bedürfnisse pflegender Angehörige einzugehen (53\% weniger gut bzw. überhaupt nicht gut). Die Möglichkeiten und Strukturen für Hausärzt*innen innerhalb des Gesundheitswesens, eine gute Unterstützung pflegender Angehöriger zu leisten, werden von $44 \%$ positiv beurteilt, von $52 \%$ negativ. Ein häufiger Kritikpunkt in der offenen Nachfrage betrifft das Fehlen von ausreichenden interprofessionellen Strukturen (z. B. Brückenpflege, stationäre Einrichtungen der Palliativversorgung) sowie bürokratische Hürden ( $\triangleright$ Tab.3).

Zum Ende der Befragung wurden die Teilnehmer*innen mit zwei Aussagen konfrontiert, die im Zuge der Vorbereitung der mehrteiligen Studie im Rahmen einer Fokusgruppe mit Hausärzt*innen artikuliert wurden. Im Sinne einer Gesamtbeurteilung zeigt sich, dass die überwiegende Mehrheit der Befragten zwar das hausärztliche Setting als primäre Anlaufstelle für die Bedürfnisse Pflegender betrachtet. Allerdings fühlen sich viele Befragte aufgrund der derzeitigen Rahmenbedingungen eingeschränkt, 
- Tab.2 Antworten zu Frage 13: „Die hausärztliche Unterstützung pflegender Angehöriger kann verschiedene Herausforderungen mit sich bringen. Bitte geben Sie jeweils an, für wie groß Sie die folgenden Herausforderungen tendenziell halten?“

\begin{tabular}{|c|c|c|c|}
\hline & $\begin{array}{l}\text { Sehr groß/ } \\
\text { Eher groß } \\
\text { Insgesamt } \\
(N=3556)\end{array}$ & $\begin{array}{l}\text { Sehr groß| } \\
\text { Eher groß } \\
\text { Urbane Ärzt*innen } \\
\text { ( } n=1780) \text { vs. Land- } \\
\text { ärzt*innen ( } n=1776)\end{array}$ & $\begin{array}{l}\text { Sehr groß| } \\
\text { Eher groß } \\
\text { Ärzt*innen mit geriatr. } \\
\text { Weiterbildung }(n=641) \\
\text { vs. ohne }(n=2915)\end{array}$ \\
\hline Frühzeitige Identifizierung und Ansprache pflegender Angehöriger & $59 \%(2098)$ & $59 \%(1778) / 59 \%(1778)$ & $44 \%(280) / 63 \%(1818)$ \\
\hline $\begin{array}{l}\text { Sicherstellung eines regelmäßigen Austauschs (z. B. weil pflegende } \\
\text { Person einen anderen Hausarzt hat und so kein automatischer } \\
\text { Gesprächsanlass besteht) }\end{array}$ & $43 \%(1528)$ & $47 \%(843) / 38 \%(685)$ & $26 \%(167) / 47 \%(1361)$ \\
\hline $\begin{array}{l}\text { Genügend Zeit für die Beratung pflegender Angehöriger zur } \\
\text { Verfügung stellen }\end{array}$ & $68 \%(2432)$ & $75 \%(1334) / 62 \%(1098)$ & $44 \%(282) / 74 \%(2150)$ \\
\hline Compliance pflegender Angehöriger sicherstellen & $10 \%(340)$ & $12 \%(205) / 8 \%(135)$ & $2 \%(11) / 11 \%(329)$ \\
\hline $\begin{array}{l}\text { Möglichst unverzögerte Einschätzung, welche konkreten Bedürfnisse } \\
\text { bzw. Probleme die pflegende Person hat }\end{array}$ & $26 \%(928)$ & $23 \%(402) / 30 \%(526)$ & $6 \%(38) / 31 \%(890)$ \\
\hline $\begin{array}{l}\text { Im Fall einer Dekompensation zeitnahe Entlastungsoptionen realisieren, } \\
\text { ohne dass dabei die Pflegesituation destabilisiert wird }\end{array}$ & $87 \%(3124)$ & $84 \%(1500) / 91 \%(1624)$ & $83 \%(532) / 89 \%(2592)$ \\
\hline Rechtliche Beratung pflegender Angehöriger & $54 \%(1896)$ & $47 \%(842) / 59 \%(1054)$ & $23 \%(145) / 60 \%(1751)$ \\
\hline $\begin{array}{l}\text { Bedürfnisse und Wünsche des Pflegenden und Gepflegten } \\
\text { gleichermaßen berücksichtigen }\end{array}$ & $44 \%(1578)$ & $46 \%(825) / 42 \%(753)$ & $12 \%(74) / 52 \%(1504)$ \\
\hline $\begin{array}{l}\text { Individuell passgenaue Unterstützungs-, Hilfs- und Entlastungsangebote } \\
\text { in der Umgebung finden }\end{array}$ & $79 \%(2800)$ & $69 \%(1225) / 88 \%(1575)$ & $54 \%(343) / 85 \%$ (2457) \\
\hline $\begin{array}{l}\text { Rasche Verfügbarkeit von Entlastungsangeboten (z. B. Kurzzeit-/ } \\
\text { Tagespflege, psychosoziale Angebote) }\end{array}$ & $89 \%(3144)$ & $83 \%$ (1483)/93\% (1661) & $74 \%$ (471)/92\% (2673) \\
\hline
\end{tabular}

- Tab.3 Antworten zu Frage 23: „Hier stehen zwei Aussagen, die von Hausärzt*innen aus einer vorangegangenen Interviewstudie stammen. Wie stehen Sie zu diesen Aussagen?“

\begin{tabular}{|c|c|c|c|}
\hline & $\begin{array}{l}\text { Stimme voll und } \\
\text { ganz zu/Stimme } \\
\text { eher zu } \\
\text { Insgesamt } \\
(\mathrm{N}=3556)\end{array}$ & $\begin{array}{l}\text { Stimme voll und ganz } \\
\text { zu/Stimme eher zu } \\
\text { Urbane Ärzt*innen } \\
\text { ( } n=1780 \text { ) vs. Land- } \\
\text { ärzt*innen ( } n=1776 \text { ) }\end{array}$ & $\begin{array}{l}\text { Stimme voll und ganz } \\
\text { zu/Stimme eher zu } \\
\text { Ärzt*innen mit geriatr. } \\
\text { Weiterbildung }(n=641 \text { ) } \\
\text { vs. ohne }(n=2915)\end{array}$ \\
\hline $\begin{array}{l}\text { „Hausärzt*innen sollten als vorrangige Anlaufstelle für die Bedürfnisse } \\
\text { pflegender Personen zuständig sein und die Pflege konsequent } \\
\text { koordinieren.“ }\end{array}$ & $77 \%(2756)$ & $70 \%(1236) / 86 \%(1520)$ & $77 \%$ (497)/77\% (2259) \\
\hline $\begin{array}{l}\text { „Ich würde gerne eine wichtigere und präsentere Rolle für pflegende } \\
\text { Angehörige spielen, kann dies aber aufgrund der derzeitigen } \\
\text { Rahmenbedingungen nicht tun.“ }\end{array}$ & $56 \%(1985)$ & $49 \%$ (879)/62\% (1106) & $54 \%(346) / 56 \%$ (1639) \\
\hline
\end{tabular}

wenn es darum geht, eine präsentere Rolle für besagte Zielgruppe einzunehmen.

In einer offenen Nachfrage artikuliert ein Teil der Befragten Wünsche nach einer besseren Einbeziehung von Hausärzt*innen in kommunale Gesundheits- und Versorgungsstrukturen bzw. eine engere Zusammenarbeit im interprofessionellen Netz, die leichtere Überblicke und Verweise ermöglichen. Zudem wird der Wunsch artikuliert, dass die Krankenkassen pflegende Angehörige systematisch und konsequent begleiten und so die hausärztliche Arbeit flankieren. Ebenfalls angeregt wird die Schaffung eines festen Begleitprogramms, in das pflegende Angehörige von Hausärzt*innen eingeschrieben werden können und das ihnen anhand einer individuellen Risikostratifizierung kontinuierliche Information und Beratung sowie bei Bedarf Interventionsmaßnahmen zusichert. 


\section{Diskussion}

\section{Zusammenfassung und Befunde anderer Studien}

Die Ergebnisse der Befragung zeigen, dass Allgemeinmediziner*innen das hausärztliche Setting als zentrale Betreuungs- und Versorgungsinstanz für pflegende Angehörige betrachten. Eine große Mehrheit der Befragten gibt an, im Praxisalltag häufiger mit pflegenden Personen zu tun zu haben, deren Zahl in den zurückliegenden Jahren gewachsen ist. Verbreitet kommen Betreuungssituationen dadurch zustande, dass pflegende Angehörige auf den/die Hausärzt* in des Gepflegten zugehen; andere Gesprächskonstellationen sind seltener. Die häufigsten Inhalte betreffen eine Verschlechterung der Pflegesituation und eine Veränderung des Pflegebedarfs; Beratungsgespräche in der Anfangs- und Vorbereitungsphase der Pflege sind dagegen vergleichsweise weniger häufig.

Während die Befragten ein komplexes Geflecht an Bedürfnissen auf Seiten pflegender Angehöriger wahrnehmen, zeigen sich zwischen urban und ländlich situierten Ärzt*innen unterschiedliche Akzente. So gewichten Landärzt ${ }^{*}$ innen eine proaktive, kollaborative und psychosoziale Betreuung stärker, wohingegen Ärzt*innen in städtischen Regionen vermehrt auf ein dort stärker vorhandenes Facharzt- und Hilfsnetzwerk setzen. Insbesondere Ärzt*innen mit geriatrischer Weiterbildung fallen dadurch auf, dass sie öfter auf Hilfs- und Beratungsakteure verweisen.

Die Abfrage verschiedener Herausforderungen hat gezeigt, dass sich eine umfassende Unterstützung pflegender Angehöriger im hausärztlichen Praxisalltag anspruchsvoll gestalten kann, v. a. mit Blick auf die Organisation zeitnaher Entlastungsangebote zur Abwendung von Versorgungskrisen, die Vermittlung zu geeigneten Hilfsangeboten oder die frühzeitige Identifizierung informeller Pflegepersonen. Auffallend ist, dass viele Herausforderungen von geriatrisch weitergebildeten Ärzt*innen in geringerem Maße wahrgenommen werden. Dies kann damit in Zusammenhang stehen, dass entsprechend weiterqualifizierte Allgemeinmediziner*innen in merklich höherem Maße die Kooperation mit interprofessionellen Akteuren für Pflegende und Gepflegte suchen und ihr Praxispersonal bei der Unterstützung pflegender Angehöriger stärker einbeziehen.

Die Befunde der Studie reflektieren insgesamt die Ergebnisse der vorangegangenen Online-Befragung [27] sowie anderer Erhebungen, wonach Hausärzt*innen für die Gruppe pflegender Angehöriger von großer Bedeutung sind [6, 20-22, 25, 33-35]. Pflegende Angehörige, aber auch Hausärzt*innen selbst messen dem primärärztlichen Setting großes Potenzial zu, die Probleme von Pflegepersonen zu adressieren [7, 14, 23, 24, 33]. So hebt eine in Irland realisierte Interviewstudie mit pflegenden Personen die prioritäre Rolle des Hausarztes hervor, wenn es darum geht, längerfristige Coping- und Resilienzstrategien in häuslichen Pflegesettings zu entwickeln [34]. Greenwood und Kolleg*innen [26] konnten ihrerseits herausarbeiten, dass das primärärztliche Setting eine zentrale Versorgungsaufgabe bei der Unterstützung spezifischer Gruppen von pflegenden Personen wahrnehmen und die weitere Versorgung effektiv koordinieren kann.

Wie auch die vorliegende Arbeit gezeigt hat, lassen sich in anderen durchgeführten Studien zum Thema Schwachpunkte der hausärztlichen Versorgung einkreisen. Qualitative Befragungsstudien lassen erkennen, dass Hausärzt"innen die Betreuung pflegender Angehöriger als einen wichtigen Teil ihrer Arbeit ansehen, jedoch in Anbetracht von Zeit- und Ressourcenknappheit nicht immer umfänglich den Bedürfnissen häuslicher Pflegesituationen gerecht werden können $[6,36]$. So nehmen Hausärzt*innen, wie etwa Arbeiten von Höppner et al. [31] zeigen, insbesondere ihren Beratungsauftrag zur Organisation der Pflege und als Informationsquelle zu Erkrankung und Verlauf wahr. Physische und psychische Belastungen der pflegenden Angehörigen werden demgegenüber eher nachrangig bemerkt oder behandelt $[21,25,26]$. Die Forschungsliteratur spricht hier die Tendenz an, pflegende Angehörige vorrangig in Bezug auf die gepflegte Person wahrzunehmen, wobei psychosoziale Auswirkungen marginalisiert werden [31, 37]. Wie diverse Arbeiten betonen, ist zur effektiven Unterstützung einer gelingenden Pflege bedeutend, in der triadischen Konstellation die Bedürfnisse und Belastungen Pflegender und Gepflegter gleichermaßen zu berücksichtigen [24-26, 33, 38].

Eine weitere Problematik, die sich in den Befragungsergebnissen widerspiegelt, betrifft die Identifizierung pflegender Personen [36]. Auch in den qualitativen Untersuchungen von Burridge et al. fällt auf, dass Pflegende sich nicht immer trauen, ihre Problematiken darzulegen, wenn Hausärzt*innen ihnen nicht signalisieren, dass sie sich als Anlaufstelle verstehen [25, 39]. Hausärzt*innen wiederum sind oftmals unsicher, in ihrer Praxis informelle Pflegepersonen als solche erkennen zu können [22]. Damit pflegende Angehörige ihre Anliegen und Probleme artikulieren, ist es wichtig, ihnen frühzeitig zu signalisieren, dass ihre Unterstützung in den hausärztlichen Zuständigkeitsbereich fällt.

Ferner sind nur einem Teil der Hausärzt*innen Leitlinien zum Thema bekannt; auch das Praxispersonal wird lediglich von einem Teil der Praxen bei der Unterstützung Pflegender einbezogen. Eine Fokusgruppenstudie von Krug et al. belegt, dass das Erkennen von Angehörigen und deren Belastungssituation durch das Praxispersonal oft nicht systematisch, sondern reaktiv auf Überforderungsverhalten erfolgt, was mit Faktoren wie Weiterbildung und Praxisorganisation zusammenhängt [31].

Die Online-Befragung von pflegenden Angehörigen [27] hat eruiert, dass diese sich stärker eine hausärztliche Rolle als Verweis- und Vermittlungsinstanz zu Hilfsangeboten wünschen. Dies korrespondiert mit den Befunden der vorliegenden Befragung, da nur ein Teil der Hausärzt*innen von konsequenten Verweisen zu unterstützenden oder entlastenden Angeboten Gebrauch macht. Diese Resultate werden z. B. von Kiceniuk et al. bestätigt, die herausarbeiten, dass der Bedarf pflegender Personen und Gepflegter nach frühzeitigen und systematischen Verweisen zu entlastenden Akteuren nicht immer von Hausärzt*innen adressiert wird. Indem pflegende Angehörige an solche Unterstützungsakteure vermittelt werden, erhalten sie rechtzeitigen Zugang zu Informationen zur Organisation der Pflege [8, 33], die einen längeren Aufenthalt der Pflegepersonen zu Hause ohne Versorgungskrisen (z. B. Hospitalisierungen) ermöglichen [19, 40]. Die nicht immer gegebene Vermittlungstätigkeit hin zu Hilfsnetzen korrespondiert mit dem Befund, dass Hausärzt*innen oft keinen ausreichenden Überblick über externe Unterstützungsformen für pflegende Personen haben [19, 24] und selten in kommunale, 
strukturierte Gesundheitsnetzwerke eingebunden sind [35, 41]. Insofern ist es entscheidend, die interdisziplinäre sektorenübergreifende Kommunikation zu stärken und (in)formelle Kooperationsnetze zu errichten.

\section{Limitationen}

Die Befragung weist mehrere Limitationen auf, die entsprechend zu reflektieren sind:

- Begrenzte Fallzahl sowie begrenzte Rücklaufquote

- Regionaler Rekrutierungsschwerpunkt in 3 Bundesländern

- Möglichkeit, dass verstärkt Hausärzt*innen an den Interviews teilgenommen haben, die online-affin sind und/oder ein thematisches Interesse haben (der hohe Anteil von Ärzt*innen mit geriatrischen Weiterbildungen ist hierauf ein Hinweis)

Dennoch lassen der gewählte Methodenansatz und die heterogene Stichprobe Aussagen zu, die in die Breite der Hausärzteschaft hineinreichen.

\section{Schlussfolgerungen}

Die Hausarztpraxis kann eine vitale Rolle bei der Unterstützung pflegender Angehöriger einnehmen und den Pflegenden somit Bestärkung und Sicherheit im Hinblick auf die Pflegeorganisation vermitteln. Ein großer Teil der befragten Hausärzt*innen legt Wert auf die Betreuung pflegender Personen und ist sich ihrer wichtigen Rolle bei der Unterstützung dieser Zielgruppe bewusst. Dennoch gibt es verschiedene Herausforderungen, die im Praxisalltag bewirken, dass Hausärzt*innen den Bedürfnissen Pflegender nicht immer umfänglich gerecht werden können [42]. Hierzu zählen die Organisation zeitnaher Entlastungsangebote zur Abwendung krisenhafter Versorgungssituationen, die Vermittlung zu geeigneten Hilfsangeboten oder die frühzeitige Identifizierung informeller Pflegepersonen.

Indem Hausärzt*innen auf die Bedürfnisse Pflegender eingehen, sind sie in der Lage, häusliche Pflegesettings längerfristig zu stabilisieren sowie einem „Ausbrennen“ der Pflegeperson vorzubeugen. Dazu sollten pflegende Angehörige früh identifiziert und angesprochen werden. Zudem ist es wichtig, in der triadischen Konstellation die Bedürfnisse, Wünsche und Belastungen Pflegender und Gepflegter gleichermaßen zu berücksichtigen.

Ein zentraler Hebel für die Effektivierung der hausärztlichen Unterstützung pflegender Angehöriger besteht neben einer aktiven Einbeziehung des Praxispersonals in einer besseren und systematischeren Verzahnung von Hausärzt*innen mit Beratungs- und Unterstützungsakteuren wie z. B. Pflegestützpunkten, ambulanten psychiatrischen Diensten und Demenz-Netzwerken. Indem Patient*innen und Angehörige rechtzeitig an derlei Hilfsnetzwerke herangeführt werden, wird die Aufrechterhaltung einer guten Lebensqualität für beide entscheidend unterstützt.

\section{KERNAUSSAGEN}

- Hausärzt*innen können für pflegende Angehörige eine zentrale Betreuungs- und Unterstützungsrolle spielen. Indem sie auf die Bedürfnisse Pflegender eingehen, sind sie in der Lage, häusliche Pflegesettings längerfristig zu stabilisieren.

- Während die Befragten ein komplexes Geflecht an Bedürfnissen auf Seiten pflegender Angehöriger wahrnehmen, zeigen sich zwischen urban und ländlich situierten Ärzt*innen unterschiedliche Akzente. Landärzt*innen gewichten eine proaktive und psychosoziale Betreuung stärker, wohingegen Ärzt*innen in städtischen Regionen vermehrt auf das Facharzt- und Hilfsnetzwerk setzen.

- Hausärzt*innen erleben im Praxisalltag diverse Herausforderungen bei der Betreuung Pflegender, darunter die zeitnahe Organisation passender Entlastungsangebote, die Vermittlung zu geeigneten Hilfsangeboten oder die frühzeitige Identifizierung informeller Pflegepersonen.

- Um die hausärztliche Rolle zu stärken, sollten Pflegende frühzeitig vom Praxisteam angesprochen und konsequent an Hilfs- und Unterstützungsangebote verwiesen werden. Zur effektiven Unterstützung einer gelingenden Pflege ist es wichtig, in der triadischen Konstellation die Bedürfnisse Pflegender und Gepflegter gleichermaßen zu berücksichtigen.

\section{Anmerkungen}

\section{Förderung}

Bei der vorliegenden Studie handelt es sich um eine eigenständige Untersuchung im weiteren inhaltlichen Kontext eines auf 3 Jahre geförderten Innovationsfonds-Modellprojektes zur ambulanten medizinisch-pflegerischen Demenzversorgung (DemStepCare, Proposal-ID: 01NVF18 027). Dabei steht die Optimierung der hausarztbasierten Demenzversorgung im Zentrum. Der Fördergeber ist weder an der Ausgestaltung der Studie noch an der Erhebung, Analyse oder Interpretation von Studiendaten beteiligt; ebenso wenig spielt er eine Rolle bei projektbezogenen Veröffentlichungen.

\section{Innovationsfonds neue Versorgungsformen: DemStepCare}

Konsortialführung: Landeskrankenhaus (AöR) mit dem Zentrum für psychische Gesundheit im Alter (ZpGA), Mainz, sowie Rhein-Mosel Akademie (RMA), Andernach

Konsortialpartner: Albert-Ludwigs-Universität Freiburg, BKK Landesverband Mitte, DAK Gesundheit, LWL Klinikum Gütersloh, Landespflegekammer Rheinland-Pfalz, MEDI Südwest $\mathrm{GmbH}$, Techniker Krankenkasse, Universitätsmedizin der Johannes-Gutenberg-Universität Mainz 


\section{Informationen zum Projekt:}

Teresa Weber

Zentrum für psychische Gesundheit im Alter (ZpGA)

Landeskrankenhaus (AöR)

Hartmühlenweg 2-4

55122 Mainz

Tel.: $06131-3783101$

E-Mail: t.weber@zpga.landeskrankenhaus.de

https://www.landeskrankenhaus.de/aktuelles/projekte-imlandeskrankenhaus/zentrum-fuer-psychische-gesundheit-imalter-zpga/demstepcare-das-hausarztbasierte-versorgungs konzept

\section{Einhaltung ethischer Richtlinien}

Das assoziierte Modellprojekt DemStepCare wurde von der Ethikkommission der Landesärztekammer Rheinland-Pfalz genehmigt (Referenznummer: 2019-14427). Alle Teilnehmer*innen (Patient*innen, Pflegepersonal, Allgemeinmediziner*innen, Fallmanager*innen) geben eine schriftliche Einwilligung nach Aufklärung, bevor Daten für die Studie erhoben werden. Wenn ein Erziehungsberechtigter vorhanden ist, muss der oder die Erziehungsberechtigte neben dem Patienten/der Patientin eine schriftliche Einwilligung erteilen. Alle Patienten benötigen zur Einwilligung eine Grundkompetenz, ansonsten können sie nicht teilnehmen.

Alle Verfahren, die in Studien mit menschlichen Teilnehmern oder an menschlichem Gewebe durchgeführt werden, entsprechen den ethischen Standards des institutionellen und/oder nationalen Forschungsausschusses und der Helsinki-Deklaration von 1975 und ihren späteren Änderungen oder vergleichbaren ethischen Standards.

\section{Interessenkonflikt}

Die Autorinnen/Autoren geben an, dass kein Interessenkonflikt besteht.

\section{Literatur}

[1] Eurostat. Bevölkerungsstruktur und Bevölkerungsalterung. https://ec.eu ropa.eu/eurostat/statistics-explained/index.php?

title=Population_structure_and_ageing/de; Stand: 13.07.2021

[2] WHO Regional Office for Europe. Home Care in Europe. Copenhagen: WHO/Europe; 2015.

[3] Statistisches Bundesamt. Pflegestatistik 2019. https://www.destatis.de/ DE/Themen/Gesellschaft-Umwelt/Gesundheit/Pflege/Publikationen/ _publikationen-innen-pflegestatistik-deutschland-ergebnisse.html; Stand: 13.07.2021

[4] Nowossadeck S, Engstler H, Klaus D. Pflege und Unterstützung durch Angehörige. Report Altersdaten 1/2016. Berlin: Deutsches Zentrum für Altersfragen. 2016

[5] Tesch-Römer C, Hagen C. Ausgewählte Aspekte zur informellen häuslichen Pflege in Deutschland. Berlin: Deutsches Zentrum für Altersfragen. 2018 https://nbn-resolving.org/urn:nbn:de:0168-ssoar-58856-1; Stand: 13.07 .2021
[6] Connell CM, Boise L, Stuckey JC et al. Attitudes toward the diagnosis and disclosure of dementia among family caregivers and primary care physicians. Gerontologist 2004; 44 (4): 500-507

[7] DAK. DAK-Pflege-Report 2015. Hamburg: DAK-Gesundheit. 2015

[8] Wuttke-Linnemann A, Henrici CB, Müller $N$ et al. Bouncing back from the burden of dementia: Predictors of resilience from the perspective of the patient, the spousal caregiver, and the dyad - An exploratory study. GeroPsych 2020; 33 (3): 170-181

[9] Klaus D, Tesch-Römer C. Pflege und Unterstützung bei gesundheitlichen Einschränkungen: Welchen Beitrag leisten Personen in der zweiten Lebenshälfte für andere? In: Mahne K, Wolff J, Simonson J, et al., Hrsg. Altern im Wandel: Zwei Jahrzehnte Deutscher Alterssurvey. Wiesbaden: Springer; 2016: 185-200

[10] Schmidt M, Schneekloth U. Abschlussbericht zur Studie „Wirkungen des Pflege-Weiterentwicklungsgesetzes“. Berlin: Bundesministerium für Gesundheit; 2011

[11] Bestmann B, Wüstholz E, Verheyen F. Belastung und sozialer Zusammenhalt. Eine Befragung zur Situation von pflegenden Angehörigen. Hamburg: Techniker Krankenkasse; 2014

[12] O'Reilly D, Connolly S, Rosato M et al. Is caring associated with an increased risk of mortality? A longitudinal study. Soc Sci Med 2008; 67 (8): 1282-1290

[13] Beach SR, Schulz R, Williamson GM et al. Risk factors for potentially harmful informal caregiver behavior. J Am Geriatr Soc 2005; 53 (2): 255-261

[14] Cherry MG, Salmon P, Dickson JM et al. Factors influencing the resilience of carers of individuals with dementia. Rev Clin Gerontol 2013; 23 (4): 251-266

[15] Schulz R, Sherwood P. Physical and mental health effects of family caregiving. Am J Nurs 2008; 108 (9): 23-27

[16] Dias R, Santos RL, Sousa MF et al. Resilience of caregivers of people with dementia: a systematic review of biological and psychosocial determinants. Trends Psychiatry Psychother 2015; 37 (1): 12-19

[17] Joling K, Windle G, Dröes RM et al. Factors of resilience in informal caregivers of people with dementia from integrative international data analysis. Dement Geriatr Cogn Disord 2016; 42 (3): 198-214

[18] Roepke SK, Mausbach BT, Patterson TL et al. Effects of Alzheimer caregiving on allostatic load. J Health Psychol 2011; 16 (1): 58-69

[19] Geschke K, Scheurich A, Schermuly I et al. Effectivity of early psychosocial counselling for family caregivers in general practitioner based dementia care. Dtsch Med Wochenschr 2012; 137 (43): 2201-2206

[20] Romero-Moreno R, Márquez-González M, Mausbach BT et al. Variables modulating depression in dementia caregivers: a longitudinal study. Int Psychogeriatr 2012; 24 (8): 1316-1324

[21] Bulsara CE, Fynn N. An exploratory study of gp awareness of carer emotional needs in Western Australia. BMC Fam Pract 2006; 7: 33

[22] Greenwood N, Mackenzie A, Habibi R et al. General practitioners and carers: a questionnaire survey of attitudes, awareness of issues, barriers and enablers to provision of services. BMC Fam Pract 2010; 11: 100

[23] Kassenärztliche Bundesvereinigung. Versichertenbefragung 2018. Ergebnisse einer repräsentativen Bevölkerungsumfrage. Berlin, 2018

[24] Laux N, Melchinger H, Scheurich A et al. Improving General Practitioners guided dementia care. Dtsch med Wochenschr 2010; 135 (44): 21752180

[25] Burridge LH, Mitchell G, Jiwa M et al. Helping lay carers of people with advanced cancer and their GPs to talk: An exploration of Australian users' views of a simple carer health checklist. Health and Social Care in the Community 2017; 25 (2): 357-365

[26] Greenwood N, Mackenzie A, Harris R et al. Perception of the role of general practice and practical support measures for carers of stroke survivors: a qualitative study. BMC Fam Pract 2011; 12: 57

[27] Wangler J, Jansky M. Support, needs and expectations of family caregivers regarding general practitioners - results from an online survey. BMC Fam Pract 2021; 22: 47 
[28] Gemeinsamer Bundesausschuss Innovationsausschuss. DemStepCare Hausarztbasierte Demenzversorgung mit koordinierter Kooperation und risikostratifiziertem Einsatz spezialisierter Pflegekräfte. https:// innovationsfonds.g-ba.de/projekte/neue-versorgungsformen/demstep care-hausarztbasierte-demenzversorgung-mit-koordinierter-kooperati on-und-risikostratifiziertem-einsatz-spezialisierter-pflegekraefte.279; Stand: 13.07.2021

[29] Bablok I, Binder H, Stelzer D et al. Primary dementia care based on the individual needs of the patient: study protocol of the cluster randomized controlled trial, DemStepCare. BMC Geriatrics 2021; 21 (1): 222

[30] Wangler J, Fellgiebel A, Jansky M. Dementia Diagnosis in General Practitioner Care - Attitudes, Procedures and Challenges from the Perspective of General Practitioners in Rhineland-Palatinate. Dtsch Med Wochenschr 2018; 143 (19): 165-171

[31] Höppner C, Schneemilch M, Lichte T. Pflegende Angehörige und ihre Belastungen in Hausarztpraxen identifizieren - Hindernisse und Empfehlungen. Z Allg Med 2015; 91: 310-313

[32] Mayring P. Qualitative Inhaltsanalyse. Grundlagen und Techniken. 11. Aufl. Weinheim: Beltz; 2010

[33] Donath C, Gräßel E, Großfeld-Schmitz M et al. Effects of general practitioner training and family support services on the care of home-dwelling dementia patients - Results of a controlled cluster-randomized study. BMC Health Serv Res 2010; 10: 314

[34] Lane P, McKenna H, Ryan A et al. The Experience of the Family Caregivers' Role: A Qualitative Study. Res Theory Nurs Pract 2003; 17 (2): 137-151
[35] Prüfer F, Joos S, Milksch A. Die Rolle des Hausarztes in der kommunalen Gesundheitsförderung. Prävention und Gesundheitsförderung 2015; 10 (2): 180-185

[36] Schneekloth U, Wahl HW. Möglichkeiten und Grenzen selbstständiger Lebensführung in privaten Haushalten. Abschlussbericht im Auftrag des Bundesministeriums für Familie, Senioren, Frauen und Jugend. München, 2005

[37] Adams WL, Mcllvain HE, Geske JA et al. Physicians' perspectives on caring for cognitively impaired elders. Gerontologist 2005; 45 (2): 231-239

[38] The Princess Royal Trust for Carers and Royal College of General Practitioners. Supporting carers: an action guide for general practitioners and their teams. London: RCGP. 2011

[39] Burridge LH, Mitchell G, Jiwa M et al. Helping lay carers of people with advanced cancer and their GPs to talk: An exploration of Australian users' views of a simple carer health checklist. Health and Social Care in the Community 2017; 25 (2): 357-365

[40] Thyrian JR, Fiss T, Dreier A et al. Life- and person-centred help in Mecklenburg-Western Pomerania, Germany (DelpHi): study protocol for a randomised controlled trial. Trials 2012; 13: 56

[41] Parmar J, Anderson S, Abbasi M et al. Family Physician's and Primary Care Team's Perspectives on Supporting Family Caregivers in Primary Care Networks. Int J Environ Res Public Health 2021; 18 (6): 3293

[42] Bedard M, Gibbons C, Lambert-Belanger A et al. Development of a tool to investigate caregiving issues from the perspective of family physicians and discussion of preliminary results. Primary Health Care Research \& Development 2014; 15 (2): 220-226 